\title{
CD19CAR-CD28zeta-4-1BB-expressing Allogeneic T Lymphocytes
}

National Cancer Institute

\section{Source}

National Cancer Institute. CD19CAR-CD28zeta-4-1BB-expressing Allogeneic T

Lymphocytes. NCl Thesaurus. Code C107242.

Allogeneic T-lymphocytes transduced with a retroviral vector expressing a chimeric antigen receptor (CAR) consisting of an anti-CD19 scFv (single chain variable frag ment) coupled to the costimulatory signaling domain CD28, the signaling domain of 4-1BB (CD137), and the zeta chain of the T-cell receptor (TCR), with potential immunomodulating and antineoplastic activities. Upon transfusion, CD19CAR-CD28 zeta4-1BB-expressing allogeneic $T$ lymphocytes directs the $T$-lymphocytes to and induces selective toxicity in CD19-expressing tumor cells. CD28, a T-cell surface-associated costimulatory molecule, is required for T-cell activation, proliferation, and survival. The 41BB co-stimulatory molecule signaling domain enhances activation and signaling after recognition of CD19. Furthermore, inclusion of the 4-1BB signaling domain may increase the antitumor activity compared to the inclusion of the CD28 costimulatory domain and TCR zeta chain alone. CD19 antigen is a B-cell specific cell surface antigen, which is expressed in all B-cell lineage malignancies. 